\title{
Correspondence to YOMEGA Trial
}

\section{Hicham El Fara ${ }^{1}$ (ID}

Published online: 10 October 2019

(C) Springer Science+Business Media, LLC, part of Springer Nature 2019

Dear Editor,

I read with interest the paper published by Robert et al. [1], reporting a randomized clinical trial comparing the outcomes of One-Anastomosis Gastric Bypass (OAGB) with Roux-en-Y gastric bypass (RYGB) at 2-year follow-up (YOMEGA trial). Despite it is an interesting topic, several aspects make the results at least questionable. First, they performed a non-inferiority trial. I think this is a wrong design, as there are 2 previous randomized clinical trials and 3 meta-analyses [2-4], reporting a superiority of OAGB in terms of weight loss and remission of comorbidities. Thus, a trial to confirm this superiority would have been more appropriate. The authors describe that patients were recruited from nine high-volume bariatric institutions in France, but they required nearly 2 years to enroll 261 patients. It seems as it would have been some kind of patient selection, which was not described in the inclusion/exclusion criteria.

Regarding the interpretation of their results, they describe a significantly greater decrease in $\mathrm{HbA1C}$ at 2 years in the OAGB group, and this difference in the decrease in HbAlc was also significant in the subgroup of participants with type 2 diabetes. In the OAGB group, the proportion of participants with type 2 diabetes (T2D) who went into complete remission was $60 \%$ ( 12 of 20 participants), whereas in the RYGB group the proportion of participants who had complete remission was $38 \%$ (six of 16) $(p=0.28)$. Based on the latter, they conclude that both techniques obtain similar remission rates of T2D (based on the results of 36 patients).

Referring to nutritional results, they reported $21 \%$ of nutritional complications (defined as at least one vitamin

Hicham El Fara

hicham.elfara@gmail.com

1 El Fara Clinic, Nahyan The First ST, 11, Al Ain, United Arab Emirates deficiency, malnutrition, anemia, or iron deficiency) after OAGB and $0 \%$ after RYGB. The most threatening deficiency would be protein malnutrition, but they did not observe significant differences in postoperative albumin or prealbumin levels. Higa et al. [5] described that $82 \%$ of the patients have some kind of nutritional deficiency after RYGB. Thus, it is difficult to believe that in this paper, they report $0 \%$.

Finally, it is surprising that $3 \%$ of patients after RYGB have esophagitis at endoscopy and $8 \%$ at esophageal biopsy, 2 years after RYGB, when this technique is considered the gold standard anti-reflux surgery for obese patients. As there is no preoperative endoscopy, discarding the mucosal status, the validity of these postoperative findings is questionable.

In conclusion, the sample size of the study is insufficient and the interpretation of the results is subjective and focused on disacrediting OAGB. Therefore, the validity of these results must be interpreted with caution.

\section{References}

1. Robert M, Espalieu P, Pelascini E, et al. Efficacy and safety of one anastomosis gastric bypass versus Roux-en-Y gastric bypass for obesity (YOMEGA): a multicentre, randomised, open-label, noninferiority trial. Lancet. 2019;393:1299-309. https://doi.org/10. 1016/S0140-6736(19)30475-1.

2. Lee WJ, Yu PJ, Wang W, et al. Laparoscopic Roux-en-Yversus minigastric bypass for the treatment of morbid obesity: a prospective randomized controlled clinical trial. Ann Surg. 2005;242:20-8.

3. Ruiz-Tovar J, Carbajo MA, Jimenez JM, et al. Long-term follow-up after sleeve gastrectomy versus Roux-en-Y gastric bypass versus one-anastomosis gastric bypass: a prospective randomized comparative study of weight loss and remission of comorbidities. Surg Endosc. 2019;33:401-10.

4. Lee WJ, Lin YH. Single-anastomosis gastric bypass (SAGB): appraisal of clinical evidence. Obes Surg. 2014;24:1749-56.

5. Higa K, Ho T, Tercero F, et al. Laparoscopic Roux-en-Y gastric bypass: 10-year follow-up. Surg Obes Relat Dis. 2011;7:516-25.

Publisher's Note Springer Nature remains neutral with regard to jurisdictional claims in published maps and institutional affiliations. 